\title{
Mapping of Loss and Delay Between IP and ATM Using Network Calculus
}

\author{
Tijani Chahed, Gérard Hébuterne, and Caroline Fayet \\ Institut National des Télécommunications \\ Telecommunication Networks and Services Dept. \\ 9 rue C. Fourier, 91011 Evry CEDEX, France \\ \{tijani.chahed, gerard.hebuterne, caroline.fayet\}@int-evry.fr
}

\begin{abstract}
End-to-end QoS requires accurate mapping of QoS classes and particularly QoS parameters between the different, heterogeneous layers and protocols present at the terminal equipment and intermediate nodes. In the IP over ATM context, both technologies offer QoS capabilities but differ in architecture, service classes and performance parameters. In this paper, we consider mapping of loss and delay between Integrated Services IP and ATM, using Network Calculus (NC), a min-plus algebra formulation. NC targets lossless systems only and is thus suitable for guaranteed services. However, as our aim is to quantify and map loss and delay in the presence of loss, we extend the theory so as to account for lossy systems too, as shall be the case for non-conformant traffic and congested systems. Loss is introduced by setting constraints on both the delay and the buffer size at the network elements. Numerical applications are used to further illustrate the mapping of loss and delay between IP and ATM.
\end{abstract}

\section{Introduction}

QoS is composed of two closely related components: a user-oriented (objective and subjective) set of QoS parameters reflecting a user QoS requirement and network-oriented (objective) bounds on the QoS parameters to satisfy the requirement 1]. A correct and accurate (qualitative and quantitative) relationship between the two components is central to the overall, end-to-end QoS performance.

The approach adopted so far 2] is to map the user-oriented QoS parameters directly into a Network Performance (NP), measured at the network layer in terms of performance parameters of significance to the network provider only. The transfer parameters have been defined for the network layer only, be it for ATM [3] or IP [45]. This undermines the various heterogeneous layers superposed at the terminal equipment as well as in intermediate nodes and the protocol stack found therein [7]. As noted in [6], QoS and its corresponding bounds on the QoS parameters should be defined for each layer and translated to the next layers.

Both IP and ATM aim to offer Qos but differ in architecture and mechanisms. QoS in ATM is based on classes which are themselves based on the 
services they offer (e.g., real-time) and traffic profiles (e.g., CBR, VBR). For IP, two QoS architectures have been defined: Integrated Services (intserv) [9] and Differentiated Services (diffserv) 10 .

Intserv is based on RSVP signaling and offers a means to a per-flow QoS in IP. Three service categories have been defined: Guaranteed (GS), ControlledLoad (CLS) and Best Effort (BE). Owing to the presence of ATM at the lower layers and at the core of networks, a framework for translation of QoS between intserv and ATM is set in [1] and the interoperation of intserv classes with ATM is investigated in 12 . However these translations are valuable for mapping IP service classes to corresponding ATM QoS classes, they do not offer quantitative nor qualitative correspondence between each QoS parameter in IP and ATM. The performance parameters and their mapping at each layer provide firm basis to map the two approaches and hence guarantee end-to-end QoS [8].

The aim of this work is precisely to map QoS parameters, namely loss and delay, between the IP and ATM layers. As far as deterministic bounds are concerned, we choose to use Network Calculus (NC) [13], based on min-plus algebra, to reach this aim. NC has been defined for lossless queuing systems only. As such, it is useful to study lossless, guaranteed services. However, as our aim is to quantify loss and delay in the presence of loss, we extend NC theory so as to account for lossy systems too, as shall be the case for non-conformant GS and CLS traffic and $\mathrm{BE}$ traffic in the presence of congestion. Let us note that in an independently different context, work on the representation of the loss process using NC has been formulated in [14, in a fashion that should not interfere with the present work.

The remainder of this paper is organized as follows. In Section 2, we set the end-to-end system where IP and ATM modules co-exist. This case may arise either at the terminal equipment or some intermediate node between IP and ATM subnetworks. In this context, we study the NC formulation for maximum delay and backlog for an intserv - GS service, its transition to an ATM system and quantify the cost of a cascade of protocol stacks as opposed to a concatenation of the two systems. In Section 3, we introduce loss in NC and specifically define the regions of loss and the bounds the formulation offers. Next, we obtain representations for loss for both the constrained-buffer and the constrained-delay cases. In Section 4, we present numerical results for mapping of loss and delay between the IP and ATM layers. Conclusion and perspectives of future work are given in Section 5 .

\section{End-to-End System}

Our focus is on systems that integrate both IP and ATM modules. This case arises either at the end equipment (Figure 1) or intermediate nodes between IP and ATM subnetworks (Figure 2). 


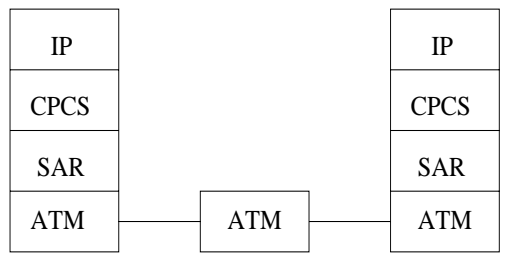

Fig. 1. End-to-end System: IP over ATM in terminal equipment

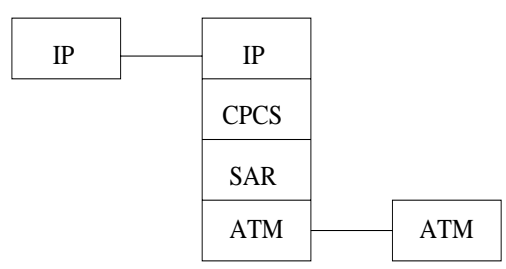

Fig. 2. End-to-end System: IP over ATM in intermediate node

\subsection{Intserv - GS}

We investigate the intserv - GS class. The arrival curve has the following Tspec (see Figure 3) as given by a token bucket and peak rate model: M (maximum packet size), $\mathrm{p}$ (peak rate), $\mathrm{r}$ (sustainable rate) and b (burst tolerance).

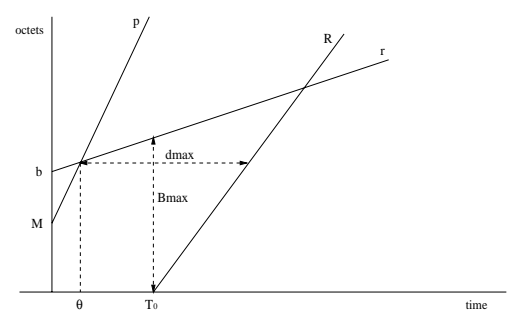

Fig. 3. Intserv - GS

Mathematically, the arrival curve is: $\alpha(t)=\min (p t+M, r t+b)$, the service curve is: $c(t)=R\left(t-T_{0}\right)^{+}$, and the output curve is: $\alpha^{*}(t)=\min \left(R\left(t-T_{0}\right), r t+b\right)$ for $t>T_{0}$. We now derive expressions for maximum delay $d_{\max }$, as implied by NC. First, we determine $\theta$. We have $r \theta+b=p \theta+M$, so, $\theta=\frac{b-M}{p-r}$. To determine $d_{\max }$, we distinguish three cases:

$$
d_{\text {max }}= \begin{cases}T_{0}+\frac{M}{r} & \text { at } t=0 \text { if } R>p \\ \frac{p-R}{R} \theta+\frac{M}{R}+T_{0} & \text { at } t=\theta \text { if } p>R>r \\ \infty & \text { if } R<r\end{cases}
$$

We now determine the maximum backlog $B_{\max }$. We distinguish two cases : 1. if $T_{0}>\theta$;

$$
B_{\text {max }}= \begin{cases}r T_{0}+b & \text { at } t=T_{0} \text { if } R>r \\ \infty & \text { if } R<r\end{cases}
$$

2. if $T_{0}<\theta$;

$$
B_{\text {max }}= \begin{cases}p T_{0}+M & \text { at } t=T_{0} \text { if } R>p \\ (p-R) \theta+M+R T_{0} & \text { at } t=\theta \text { if } p>R>r \\ \infty & \text { if } R<r\end{cases}
$$




\subsection{Transition}

The transition from one system to the next, needs to translate the Tspec of intserv - GS to appropriate, corresponding ATM rtVBR's pair of leaky buckets formulation, taking into account the overhead ratio and its implications between the layers, as shown in Figure 4 when the two systems are in cascade.

The overhead ratio $O_{i}$ is the ratio of the (expected) frame length $L_{i}$ at layer $i$ to the (expected) frame length $L_{i-1}$ at the previous, adjacent layer $i-1$ (following the direction of flow), and indicates the fractional amount of traffic that is being added (subtracted) as traffic is flowing down (up) the layers at the transmitter (receiver). Formally, at the receiver, $O_{i}=\frac{L_{i}}{n_{i-1} \times L_{i-1}}$, where $n_{i}$ is the number of segments resulting from segmentation of the frame at layer $i[8] . O_{i}$ is larger than 1 at the sender. An inverse relation governs the receiver, where $O_{i}$ is smaller than 1 .

For a system $i$ with Tspec $\left(M_{i}, p_{i}, r_{i}, b_{i}\right)$ and service components $\left(R_{i}, T_{0, i}\right)$, the following transition rules hold for $i>0$ : i. $M_{i+1}=O_{i}$, ii. $b_{i+1}=b_{i} \times O_{i}$, iii. $r_{i+1}=r_{i} \times O_{i}$ and iv. $p_{i+1}=R_{i}$. The service curve itself needs to account for the overhead ratio as the capacity at each layer needs to be accurately translated from the link layer capacity. Hence, $R_{i+1}=R_{i} \times O_{i}$.

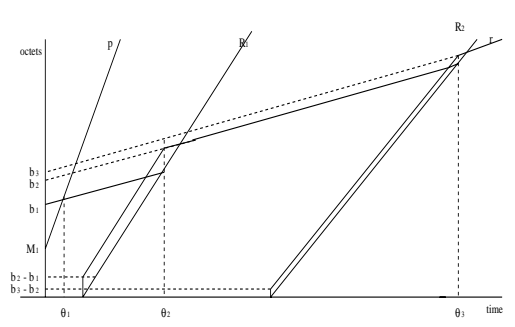

Fig. 4. Cascade

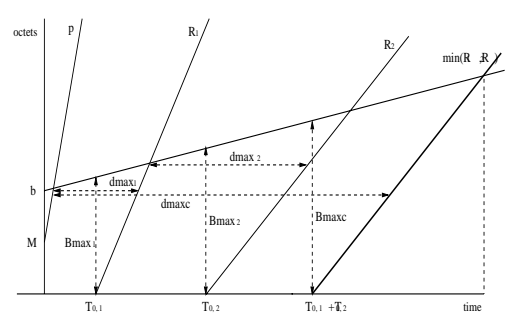

Fig. 5. Concatenation

\subsection{Next System}

Based on the NC formulation for system $i$ and the rules for transition, we develop the formulae for system $i+1$.

For a traffic descriptor : $R_{i+1}$ (peak rate), $r_{i+1}$ (sustainable rate), $M_{i+1}$ and $b_{i+1}$, where $R_{i+1}=R_{i} O_{i}, r_{i+1}=r_{i}, M_{i+1}=-R_{i} T_{0, i} O_{i}$ and $b_{i+1}=b_{i} O_{i}$, the input curve is: $\alpha_{i+1}(t)=\min \left(p_{i+1} t+M_{i+1}, r_{i+1} t+b_{i+1}\right)$ for $t>T_{0, i}$, the service curve is: $c_{i+1}(t)=R_{i+1}\left(t-T_{0, i+1}\right)^{+}$, and the output curve : $\alpha_{i+1}^{*}(t)=$ $\min \left(p_{i+2} t+M_{i+2}, r_{i+2} t+b_{i+2}\right)$ for $t>T_{0, i+1}$, where the above-mentioned transitions hold. For $\theta_{i+1}=\frac{b_{i+1}-M_{i+1}}{p_{i+1}-r_{i+1}}$, the same quantities derived in Section 2.1. for $d_{\max }$ and $B_{\max }$ are recovered. 


\subsection{Concatenation}

To have an indication on the cost of the superposition of the IP over ATM protocol stack, the cascade of the several layers may be replaced by a concatenation that acts as a single network element. One possible concatenation scenario is a network element with a service curve $c(t)=R_{\min }\left(t-T_{0, \text { sum }}\right)^{+}$where $R_{\text {min }}=\min \left(R 1, R 2, . ., R_{n}\right)$ and $T_{0, \text { sum }}=\sum_{i}^{n} T_{0, i}$, as shown in Figure 5 .

For an arrival curve $\alpha(t)=\min (p t+M, r t+b)$, the output curve is: $\alpha^{*}(t)=$ $\min \left(R_{\min } t+M_{n}, r t+b_{n}\right)$ for $t>T_{0, n}$ where $M_{n}=M \Pi_{i}^{n-1} O_{i}$ and $b_{n}=b \Pi_{i}^{n-1} O_{i}$. $\theta_{\text {concat }}$ is again equal to $\frac{b-M}{p-r}$. For $p>R_{n}>r, d_{\max }=\frac{p-R_{\min }}{R_{\min }} \theta_{\text {concat }}+\frac{M_{n}}{R_{\min }}+$ $T_{0, \text { sum }}$ at $t=\theta_{\text {concat }}$, and $B_{\max }=$

$$
\begin{cases}b+r T_{0, \text { sum }} & \text { at } t=T_{0, \text { sum }} \text { if } T_{0, \text { sum }}>\theta_{\text {concat }} \\ p \theta_{\text {concat }}+M_{n}-R_{\text {min }}\left(\theta_{\text {concat }}-T_{0, \text { sum }}\right) & \text { at } t=\theta_{\text {concat }} \text { if } T_{0, \text { sum }}<\theta_{\text {concat }}\end{cases}
$$

\section{Loss}

We now introduce loss in NC. We consider the case of a greedy source, i.e., a source with an input function equal to the arrival curve. In addition to its relative simplicity, this case offers interesting insights into worst-case analysis.

\subsection{Intervals of Loss}

Consider one system. Let us recall that the arrival curve is: $\alpha(t)=\min (p t+$ $M, r t+b)$ for $t>0$ and the service curve is: $c(t)=R\left(t-T_{0}\right)^{+}$. In case of no loss, the output curve is: $\alpha^{*}(t)=\min \left(R\left(t-T_{0}\right), r t+b\right)$ for $t>T_{0}$.

Let $B h(t)$ denote the backlog at time $t$ in a hypothetical infinite capacity queue with the same arrival and service curves as above. $B h(t)=\min (0, \alpha(t)-$ $c(t))$. Loss takes place when $B h(t)$ exceeds the actual, finite buffer capacity.

The interval of loss corresponds to all $t$ where loss occurs, and an upper bound on the number of lost packets at time $t$ in the interval of loss is the difference between $B h(t)$ and the actual buffer size, as suggested by [18]. A detailed derivation is found in Sections 3.3 and 3.4.

\subsection{Bounds on Loss}

Theorem 2 of 15 states that, under appropriate assumptions, for a flow with input function $R(t)$, constrained by an arrival curve $\alpha$, a service curve $\beta$ and an output function $R^{*}(t)$, the backlog $R(t)-R^{*}(t)$ satisfies for all $t: R(t)-$ $R^{*}(t) \leq \sup _{s \geq 0}(\alpha(s)-\beta(s))$. A greedy source is one for which $R(t)=\alpha(t)$. Let $B h_{\max }$ be the maximum backlog of a hypothetical infinite capacity queue, i.e. $B h_{\max }=\sup _{s \geq 0}(\alpha(s)-\beta(s))$.

Now, in the loss interval, for an input function $R_{l}(t)$, constrained by a an arrival curve $\alpha_{l}$, a service curve $\beta$ and an output function $R_{l}^{*}(t)$, the backlog $B l(t)=R_{l}(t)-R_{l}^{*}(t)$ satisfies for all $t: R_{l}(t)-R_{l}^{*}(t) \leq \sup _{s \geq 0}\left(\alpha_{l}(s)-\beta(s)\right)$. 
In this case, $B l_{\text {max }}=\sup _{s \geq 0}\left(\alpha_{l}(s)-\beta(s)\right)$. At a time of loss $t$, an upper bound on loss is $B h(t)-B l(t)$. An upper bound on the lost volume at a single point of time of all the interval of loss is $B h_{\max }-B l_{\max }$.

Moreover, we introduce a probability-of-loss quotient with an upper-bound equal to $\frac{B h_{\max }-B l_{\max }}{B h_{\max }}$. If the loss pattern is not cyclic, loss happens only once and the probability of loss tends to zero thereafter. However, this term obeys to the same cyclic nature as the $B_{\max }$ and $d_{\max }$ terms of NC.

There are two approaches to loss. Loss may either be caused by constraints on the buffer size or due to constraints on the delay.

\subsection{Constrained Buffer Size}

Loss Let $B_{l}\left(0<B_{l}<B_{\max }\right)$ be the buffer size at the ATM switch. Schematically, the idea is to move the line $y=B_{l}$ along the $\mathrm{y}$-axis.

Let $\left(\theta_{l}, b_{l}\right)$ be the point in time where loss occurs for the first time.

$$
\left(\theta_{l}, b_{l}\right)= \begin{cases}\left(\frac{B_{l}-b}{r}, B_{l}\right) & \text { if } B_{l}>b \\ \left(\frac{B_{l}-M}{p}, B_{l}\right) & \text { if } M<B_{l}<b \\ \left(0, B_{l}\right) & \text { if } B_{l}<M\end{cases}
$$

Let $\left(b_{e}, \theta_{e}\right)$ be the point in time where loss occurs for the last time. We distinguish two cases :

1. if $\theta<T_{0}$ (see Figure 6), $\theta_{e}=T_{0}$ and $b_{e}=B_{l}$ and

Instantaneous Loss $(\mathrm{t})=\frac{\min (p t+M, r t+b)-B_{l}}{\min (p t+M, r t+b)}$ for $\theta_{l}<t<T_{0}$ and 0 otherwise.

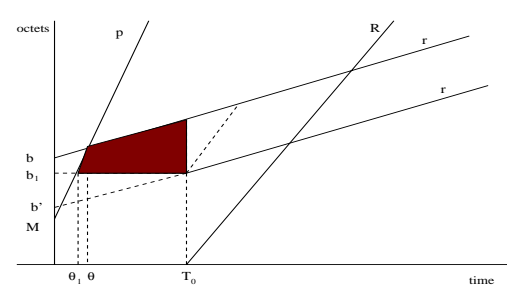

Fig. 6. Buffer-constrained Loss $-T 0>\theta \quad$ Fig. 7. Buffer-constrained Loss $-T 0<\theta$

2. if $\theta>T_{0}$ (see Figure 7), $\theta_{e}=\theta$ and $b_{e}=r \theta+b^{\prime}$ where $b^{\prime}=b-\left(r \theta_{e}+b-\right.$ $\left.R\left(\theta_{e}-T_{0}\right)-B_{l}\right)$.

$$
\text { Instantaneous Loss }(\mathrm{t})= \begin{cases}\frac{p t+M-B_{l}}{p t+M} & \text { for } t \in\left[\theta_{l}, T_{0}\right) \\ \frac{p t+M-R\left(t-T_{0}\right)-B_{l}}{p t+M} & \text { for } t \in\left[T_{0}, \theta\right]\end{cases}
$$

In general,

$$
\text { Inst. Loss }(\mathrm{t})=\frac{\min (p t+M, r t+b)-\max \left(B_{l}, R\left(t-T_{0}\right)+B_{l}\right)}{\min (p t+M, r t+b)}
$$

for $\theta_{l}<t<\max \left(T_{0}, \theta\right)$. 
System Parameters The arrival curve is : $\alpha(t)=\min (p t+M, r t+b)$ for $t>0$. The output curve is : $\alpha(t)^{*}=\min \left(r t+b^{\prime}, R\left(t-T_{0}\right)\right)$ for $t>T_{0}$, where $b^{\prime}=b-\left(r \theta_{e}+b-R\left(\theta_{e}-T_{0}\right)-B_{l}\right)$.

Maximum Backlog $B_{\max }=B_{l}$.

\section{Maximum Delay}

$$
d_{\text {max }}=\left\{\begin{array}{lll}
\frac{p-R}{R} \theta+\frac{M}{R}+T_{0} & \text { at } t=\theta & \text { if } B_{l}>b \\
\frac{p-R}{R} \theta_{l}+\frac{M}{R}+T_{0} & \text { at } t=\theta_{l} & \text { if } B_{l}<b
\end{array}\right.
$$

\subsection{Constrained Delay}

There are two approaches to loss caused by constrained-delay. Traffic exceeding the delay constraint is discarded (lost) after service or before entering the buffer.

Loss Let $d_{l}\left(0<d_{l}<d_{\max }\right)$ be the maximum delay tolerated at the ATM switch. Schematically, the idea is to vary the line $R\left(t+d_{l}-T_{0}\right)$ along the time axis.

Let $\left(\theta_{l}, b_{l}\right)$ be the point in time where loss occurs for the first time. We distinguish three cases (see Figures 8,9 and 10):

$$
\left(\theta_{l}, b_{l}\right)= \begin{cases}\left(T_{0}-d_{l}, R\left(\theta_{l}+d_{l}-T_{0}\right)\right) & \text { if } d_{l}<T_{0} \\ \left(\max \left(0, T_{0}-d_{l}\right), R\left(\theta_{l}+d_{l}-T_{0}\right)\right) & \text { if } T_{0}<d_{l}<\frac{M}{R}+T_{0} \\ \left(\frac{R\left(d_{l}-T_{0}\right)-M}{p-R}, R\left(\theta_{l}+d_{l}-T_{0}\right)\right) & \text { if } d_{l}>\frac{M}{R}+T_{0}\end{cases}
$$

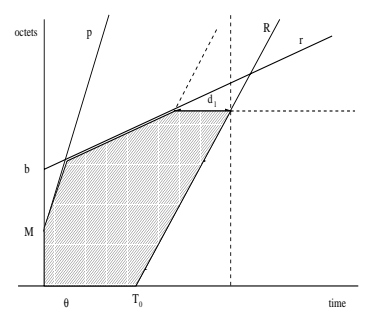

Fig. 8. Delay-constrained Loss $-d_{l}<T_{0}$

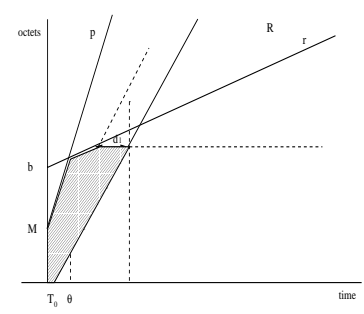

Fig. 9. Buffer-constrained Loss $-T_{0}<d_{l}<\frac{M}{R}+$ $T_{0}$

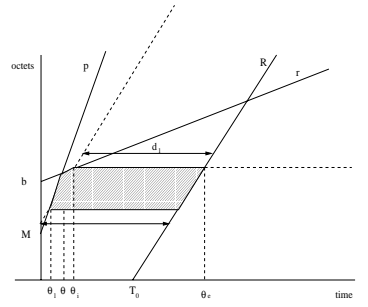

Fig. 10. Buffer-constrained Loss $-d_{l}>\frac{M}{R}+T_{0}$

Let $\left(\theta_{i}, b_{i}\right)$ be the point in time where first octet is not lost for the first time. It is given as the intersection of $y=R\left(t+d_{l}-T_{0}\right)$ and $y=r t+b$.

So, $\theta_{i}=\frac{b-R\left(d_{l}-T_{0}\right)}{R-r}$ and $b_{i}=R\left(\theta_{i}+d_{l}-T_{0}\right)$

Let $\left(\theta_{e}, b_{e}\right)$ be the point in time where loss occurs for the last time. It is given as the intersection of $y=R\left(t-T_{0}\right)$ and $y=b_{i}$. 
So, $\theta_{e}=\frac{d_{l}}{R}+T_{0}$ and $b_{e}=R\left(\theta_{e}-T_{0}\right)$

Now, for $d_{l}<\frac{M}{R}+T_{0}$,

$$
\text { Inst. Loss }(\mathrm{t})= \begin{cases}1 & \text { if } t \in\left[\theta_{l}, \theta_{i}\right) \\ \frac{b_{e}-\max \left(0, R\left(t-T_{0}\right)\right)}{r t+b-\max \left(0, R\left(t-T_{0}\right)\right)} & \text { if } t \in\left[\theta_{i}, \theta_{e}\right]\end{cases}
$$

For $d_{l}>\frac{M}{R}+T_{0}$,

$$
\text { Inst. Loss }(\mathrm{t})= \begin{cases}\frac{\min (p t+M, r t+b)-b_{l}}{\min (p t+M, r t+b)-\max \left(0, R\left(t-T_{0}\right)\right)} & \text { if } t \in\left[\theta_{l}, \theta_{i}\right) \\ \frac{b_{e}-b_{l}}{r t+b-\max \left(0, R\left(t-T_{0}\right)\right)} & \text { if } t \in\left[\theta_{i}, \theta_{e}\right]\end{cases}
$$

System Parameters In the case of delay-constrained loss, packets (or cells) that exceed the delay, may either be discarded at the entrance of the buffer or after service. We consider both cases :

1. Loss After Service

The arrival curve $\alpha(t)$ is thus: $\alpha(t)=\min (p t+M, r t+b)$. The output curve is: $\alpha^{*}(t)=\min \left(r t+b, R\left(t-T_{0}\right)\right)$ for $t>T_{0}$.

The output curve (corresponding to non lost traffic) is:

$$
\alpha^{*}(t)= \begin{cases}R\left(t-T_{0}\right) & \text { for } T_{0}<t<\theta_{l}+d_{l} \\ \min \left(r t+b, R\left(t-T_{0}\right)-b_{l}\right) & \text { for } t>\theta_{e}\end{cases}
$$

2. Loss Before Service

The arrival curve $\alpha(t)$ in this case is:

$$
\alpha(t)=\left\{\begin{array}{l}
p t+M \text { for } 0<t<\theta_{l} \\
r t+b \text { for } t>\theta_{i}
\end{array}\right.
$$

The output curve (corresponding to non lost traffic as traffic is lost before service) :

$$
\alpha^{*}(t)= \begin{cases}R\left(t-T_{0}\right) & \text { for } T_{0}<t<\theta_{l}+d_{l} \\ \min \left(r t+b, R\left(t-T_{0}\right)-b_{l}\right) & \text { for } t>\theta_{e}\end{cases}
$$

\section{Maximum Backlog}

$$
B_{\max }= \begin{cases}r t+b-R\left(t-T_{0}\right) & \text { at } t=\theta_{e} \text { if } d_{l}<\frac{M}{R}+T_{0} \\ b_{l} & \text { at } t=T_{0} \text { if } T_{0}>\theta \text { and } d_{l}>\frac{M}{R}+T_{0} \\ b_{l}-R\left(\theta-T_{0}\right) & \text { at } t=\theta \text { if } T_{0}<\theta \text { and } d_{l}>\frac{M}{R}+T_{0}\end{cases}
$$

Maximum Delay $d_{\max }=d_{l}$.

\subsection{Note on Packet Discard Mechanisms}

Loss of some ATM cells leads to loss of IP packets, depending on the discard mechanism, be it Partial Packet Discard (PPD), Early Packet Discard (EPD) [16] or ATM-version of (Random Early Discard) RED [17. In the case of PPD, for instance, loss of at least one ATM cell results in loss of the whole IP packet to which they belong. PPD is used in our numerical simulations, considered next. 


\section{Numerical Application}

\subsection{System Parameters}

We consider the end-to-end system as shown in Figure 1. Let the source have the following characteristics: $\mathrm{M}=9180$ octet; $\mathrm{b}=18360$ octet; $\mathrm{p}=180 \mathrm{Mbps}$; $\mathrm{r}=100 \mathrm{Mbps}$. Let $C_{0}=155 \mathrm{Mbps}$ and $T_{0}=0$ with a propagation delay before and after the ATM switch corresponding to 0.01 time units.

For each layer, Table 1 summarizes the length of the SDU found in each layer as well as the overhead the layer introduces, in terms of header, trailer and/or padding. $R_{i}$ is the link capacity available to each flow, on the basis of $155 \mathrm{Mbps}$ and taking into consideration the overhead ratio, at each layer $i$.

Let us note that in the following results, $B_{\max }, d_{\max }$ and the loss ratios are given in octet, normalized to the length of frames at each layer.

\subsection{Buffer-Constrained Performance}

Let $B_{l}=1000000 / 53 \approx 18861$ cells at the central ATM switch.

Figure 11 shows the maximum backlog for both the non-constrained case, i.e., no loss, (curve 'B') and the buffer-constrained case (curve 'BB'). The maximum backlog at each network element or layer, as well as its mapping, indicates the dimensioning of the buffer size to be provisioned at each subsystem in order to guarantee a no loss or a constrained loss performance.

Figure 12 shows the maximum delay at each system component for both the non-constrained case, i.e., no loss, (curve 'd') and the buffer-constrained case (curve 'dB'). This yields the mapping of delay between the different layers of the model.

Figure 13 shows the loss ratio resulting from the buffer constraint at the central ATM switch (System 4) and its mapping to the next layers.

The concatenation of all the components is as follows. In case of no loss, $R=139.83$ and $T_{0}=0.09 ; d_{\max }=0.09$ and $B_{\max }=9183600.0$. The cumulative

Table 1. Overhead Ratio and Translation of Capacity Between Layers

\begin{tabular}{lllll}
\hline \multirow{2}{*}{ System } & Layer $i$ & Length $i$ & $O_{i}$ & $R_{i}$ \\
\hline 0 & IP & 9180 & 1.020 & 171.817 \\
1 & CPCS & 9216 & 1.004 & 171.146 \\
2 & SAR & 48 & 1.000 & 171.146 \\
3 & ATM & 53 & 1.104 & 155.000 \\
4 & ATM & 53 & 1.000 & 155.000 \\
5 & ATM & 53 & 1.000 & 155.000 \\
6 & SAR & 48 & 0.906 & 140.377 \\
7 & CPCS & 9216 & 1.000 & 140.377 \\
8 & IP & 9180 & 0.996 & 139.829 \\
\hline
\end{tabular}




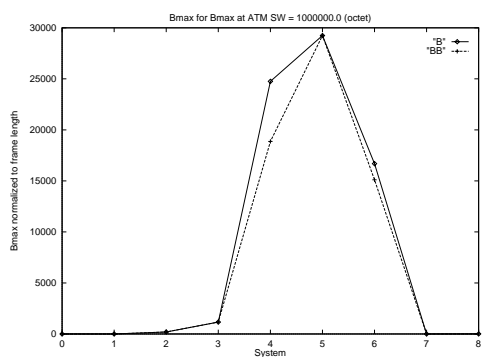

Fig. 11. Unconstrained vs. bufferconstrained maximum backlog

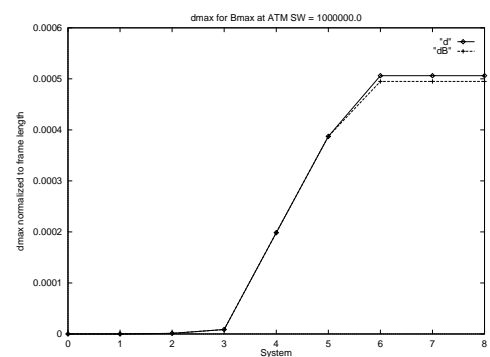

Fig. 12. Unconstrained vs bufferconstrained maximum delay

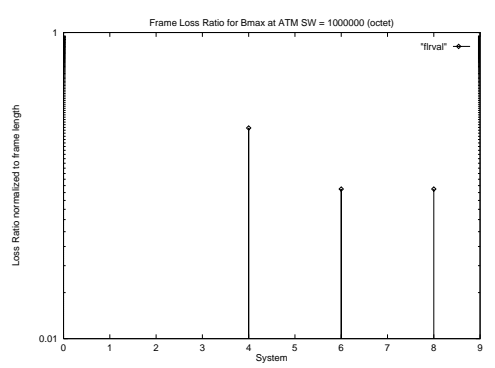

Fig. 13. Buffer-constrained loss ratio

values are $d_{\max }=0.12$ and $B_{\max }=12955812.59$. In case of loss, $d_{\max }=0.09$ and $B_{\max }=9183600.0$. The cumulative values are $d_{\max }=0.12$ and $B_{\max }=$ 12939323.4 .

\subsection{Delay-Constrained Performance}

Let $d_{l}=0.005 / 53 \approx 9.43 \times 10^{-05}$ at the central ATM switch.

Figure 14 shows the maximum backlog for both the non-constrained case (curve 'B') and the delay-constrained case (curve 'Bd'). This again indicates the buffer needed at each subsystem to have no loss or to limit loss to a given value.

Figure 15 shows the maximum delay at each system component for both the non-constrained case (curve 'd') and the delay-constrained case (curve 'dB'), yielding the mapping of delay between the different layers of the model.

Figure [16] shows the loss ratio resulting from the delay constraint at the central ATM switch (System 4) and its mapping to the next layers.

The concatenation of all the components has the following parameters: In case of no loss, we have the same values as above. In case of loss, $R=139.83$ and $T_{0}=0.11 ; d_{\max }=0.11$ and $B_{\max }=11027482.16$. The cumulative values are $d_{\max }=0.14$ and $B_{\max }=14595026.77$. 


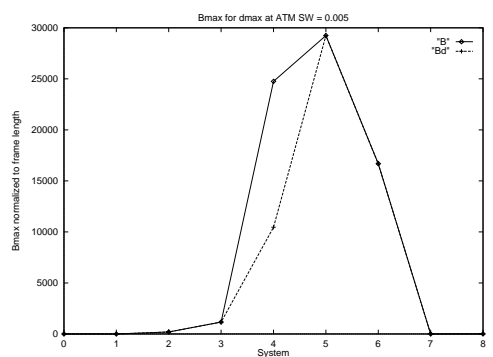

Fig. 14. Unconstrained vs delayconstrained maximum backlog

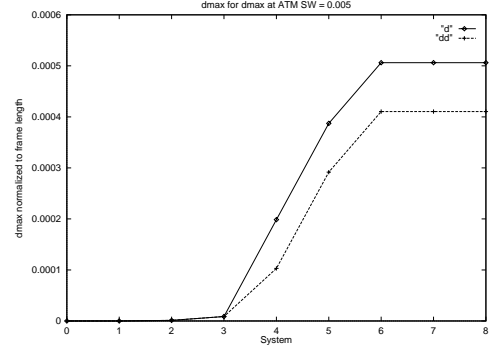

Fig. 15. Unconstrained vs delayconstrained maximum delay

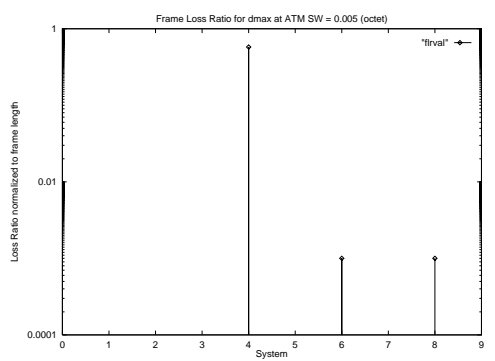

Fig. 16. Delay-constrained loss ratio

\section{Conclusion}

In this work, we mapped loss and delay between single intserv IP flows and ATM, using NC, a min-plus algebra formulation. We specifically determined the maximum backlog and delay in the system components, their transition to a next layer as well as their formulation therein. As NC only applies to lossless systems, we extended the theory and introduced loss in two fashions: either by setting constraints on the buffer size or on the delay.

This work may be further extended to cover: i. the case of three IP QoS classes into one ATM central switch, i.e. intserv over ATM and ii. the case of three intserv IP classes into one IP class, i.e. intserv as a customer to diffserv, which consists mainly of investigating the mapping of QoS between a single flow versus an aggregation of flows.

\section{References}

1. ISO/ITU-T, Quality of Service Framework, International Standard, December 1998.

2. ITU-T Recommendation I.350, General Aspects of Quality of Service and Network Performance in Digital Networks, including ISDNs, March, 1993.

3. ITU-T Recommendation I.356, -ISDN ATM Layer Cell Transfer Performances, October, 1996. 
4. ITU-T Recommendation I.380, Internet Protocol (IP) Data Communication Service - IP Packet Transfer and Availability Performance Parameters, March, 1998.

5. V. Paxson, G. Almes, J. Mahdavi, M. Mathis, RFC 2330, Framework for IP Performance Metrics, June, 1998.

6. Jae-Il Jung, Quality of Service in Telecommunications Part I: Proposition of a QoS Framework and Its Applications to B-ISDN, IEEE Communications Magazine, August, 1996.

7. T. Chahed, S. Ben Fredj, C. Fayet, Native ATM versus IP over ATM Solutions : Comparative Studies, IEEE ATM'99 Workshop, Kochi City, May 1999.

8. T. Chahed, C. Fayet, G. Hébuterne, Framework for Translation of QoS and Performance Parameters between ATM and IP, ISCC'99, Red Sea, July 1999.

9. S. Shenker, J. Wroclawski, RFC 2215, General Characterization Parameters for Integrated Service Network Elements, September 1997.

Specification of Guaranteed Quality of Service, September 1997.

Controlled-Load Network Element Service, September 1997.

10. S. Blake, D. Black, M. Carlson, E. Davies, Z. Wang, W. Weiss, RFC 2475, An Architecture for Differentiated Services, December 1998.

11. E. Crawley, L. Berger, S. Berson, F. Baker, M. Borden, J. Kraw RFC 2382, A Framework for Integrated Services and RSVP over ATM, August 1998.

12. M. Garrett, M. Borden, RFC 2381, Interoperation of Controlled-Load Service and Guaranteed Service with ATM, August 1998.

13. J-Y. Le Boudec, Application of Network Calculus To Guaranteed Service Networks, IEEE Trans on Information theory, (44) 3, May 1998.

14. J-Y. Le Boudec, P. Thiran, Network Calculus viewed as a Min-Plus System Theory, SSC research report SSC/1998/016, EPFL, 1998.

15. R. L. Cruz, Quality of Service Guarantees in Virtual Circuit Switched Networks, IEEE JSAC, 1995.

16. A. Romanow, S. Floyd, Dynamics of TCP Traffic over ATM Networks, IEEE JSAC, V. 13 N. 4, May 1995, p. 633-641.

17. O. Elloumi, H. Afifi, Improving RED Algorithm Performance in ATM, IEEE Globecom'97, Phoenix, November 1997.

18. J. Roberts, U. Mocci, J. Virtamo (Eds), Broadband Network Teletraffic, Final Report of Action COST 242, Springer, 1996. 